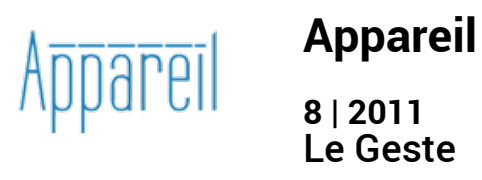

\title{
L'art-thérapie ou une pratique du geste comme un art des poupées russes
}

\section{Carole Madelaine-Dupuich}

\section{(2) OpenEdition}

\section{Journals}

Édition électronique

URL : http://journals.openedition.org/appareil/1335

DOI : 10.4000/appareil.1335

ISSN : 2101-0714

Éditeur

MSH Paris Nord

\section{Référence électronique}

Carole Madelaine-Dupuich, « L'art-thérapie ou une pratique du geste comme un art des poupées russes », Appareil [En ligne], 8 | 2011, mis en ligne le 04 novembre 2011, consulté le 30 juillet 2020. URL : http://journals.openedition.org/appareil/1335; DOI : https://doi.org/10.4000/appareil.1335

Ce document a été généré automatiquement le 30 juillet 2020.

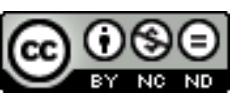

Appareil est mis à disposition selon les termes de la Licence Creative Commons Attribution - Pas d'Utilisation Commerciale - Pas de Modification 4.0 International. 


\title{
L'art-thérapie ou une pratique du geste comme un art des poupées
}

\section{russes}

\author{
Carole Madelaine-Dupuich
}

1 L'art-thérapie, art et thérapie, que sous-tendent leur association et leur influence mutuelle? Thérapie signifie non pas guérir mais prendre soin. L'art-thérapie est une méthode pratique d'accompagnement, une façon de prendre soin. Écouter et permettre un certain type de langage. Avoir l'art et la manière d'accompagner. Associé au prendre soin, l'art s'avère être une technique plutôt qu'un domaine, un moyen plutôt qu'une finalité. L'art, en cela, ouvre le déploiement d'une méthode, dont l'enjeu est de manifester la subjectivité de la personne et d'avoir lieu en développant un langage.

Découvrir un langage. Manifester son propre langage relève de techniques, d'un art. Langage et technique sont indissociables et prennent racine dans le corps. L'artthérapie accorde toute son importance au corps en le considérant comme le pivot central de sa pratique. Le corps est cette réalité matérielle, cette matière vivante, animée, douée d'énergie. Entendons par corps, cette entité faite d'esprit et de chair, d'énergie et de matière, qui est à l'origine de toutes techniques. Le corps est alors ce par quoi le langage se manifeste. Grammatisé à travers les processus techniques d'écriture et de lecture, le langage est un système reproductible développant du sens tant pour l'individu que pour le collectif. Il se rattache à la mémoire. Enfoui, le langage y est présent sans être apparent. La pratique de l'art-thérapie s'exerce alors dans le champ sensible du langage pour accéder à sa formation latente. L'art-thérapie est une méthode permettant de manifester un langage d'inconscient au moyen d'expressions du corps.

3 À partir de la grammatisation ${ }^{1}$, l'inconscient peut être considéré comme un lieu de formation, produisant des grammes non manifestes, latents, mais bien présents. L'inconscient est une latence. Et la reproductibilité, spécificité de la grammatisation, permettant la lecture des grammes sur laquelle se base le langage, révèle que l'inconscient est une mémoire. Mais la lecture ne peut avoir lieu qu'à partir d'une 
écriture, d'une inscription. Latence et mémoire définissent l'inconscient comme un système mnémotechnique capable d'écriture, capable de former des grammes équivalents à des potentiels à révéler et à manifester. Entre inconscient et art, entre mémoire, latence et technique, le lien s'avère être l'écriture en tant que tracé. Corrélativement l'art-thérapie va chercher l'écriture au moyen du corps, puisqu'il se situe à l'origine des techniques, en leur processus.

4 Étant située dans le champ du manifeste, l'art-thérapie va développer, par des expressions du corps, un langage, dont le sens de l'écriture découle d'une mise en œuvre et d'une coordination de gestes ${ }^{2}$. Seulement, l'écriture, comme système mnémotechnique, appartient aussi à l'inconscient. Elle passe par des impressions du corps, relevant d'une action indépendante de son geste, et constitue un langage dont le sens latent demande à être traduit, notamment grâce à l'analyse. Une partie du travail art-thérapeutique est justement de lire, de décortiquer un rébus, de chercher l'anagramme à partir de ce qui a été observé sensiblement dans l'écriture du geste. Le corps donne lieu à des langages manifestes ou latents qu'il place dans les champs du savoir-faire, de la saveur et du savoir définis comme des systèmes mnémotechniques. Chacun d'eux détient un inconscient, réduit à un lieu et un fonctionnement de mémoire relevant d'un processus d'écriture. Le corps permet d'emboîter l'ensemble en produisant un certain type commun de langage.

Interroger ce type de langage consiste à revenir au gramme même de l'écriture. Le gramme est une forme définie produite par la pose d'une action sur de la matière. La forme relève de la force de l'action sur la plasticité de la matière. Elle est une trace en lien direct avec l'acte dont elle provient. Le type de langage que le corps produit, son gramme, est de l'ordre de l'index ${ }^{3}$, où le sens initial pose la définition de la forme intrinsèquement comme contenant et contenu. L'accompagnement du travail artthérapeutique est d'aider la personne à rechercher la forme définie du gramme par l'exercice de son corps, par l'expression de son geste, de faire lieu à chaque instant où l'action rencontre la matière dans les différents systèmes mnémotechniques et de jouer avec, en cherchant à les emboîter.

\section{Du corps et des systèmes mnémotechniques d'écriture}

6 Le corps est à l'origine de toutes techniques, car il est en lui-même doué de techniques ${ }^{4}$. Capable de générer de l'énergie, le corps produit de l'action, des actions, à travers l'expression de ses gestes. La technique se décline alors du simple geste à l'outil, dont le point commun est d'utiliser l'action pour la faire aboutir. Marcher est une technique du corps qui utilise l'action des jambes. Parler utilise le geste de la langue dans la bouche. La parole est initialement un geste, dont l'organe de la langue est l'outil.

7 L'outil peut aussi être un objet technique extérieur au corps prolongeant son action par la force ou la précision ${ }^{5}$. En prolongeant l'action du geste de la main, le marteau permet d'enfoncer le clou, la clef d'ouvrir la porte. L'art assimilé aux techniques de l'outil utilise le corps. Il l'organise pour répondre à une action. Cet art se situe dans le champ du savoir-faire et fait appel à une grammatisation de gestes, dont le langage passe par l'expression du corps. Le savoir-faire, comme système mnémotechnique, place le corps dans le sensible, en faisant apparaître des caractères définis par des formes 
perceptibles. De ce fait, le savoir-faire, en l'ensemble de ses techniques d'outil, est observable dans la maîtrise ou la pratique de tout art.

Dans le sensible, chaque geste renvoie à percuter, saisir, façonner ${ }^{6}$ la matière. D'une manière ou d'une autre, chaque geste qui relie le corps au sensible délivre un même type, une forme, provenant d'un tact de l'action sur la matière. Chaque geste pose une forme plus ou moins évidente, selon la force de l'action et/ou la plasticité de la matière. Par les techniques de l'outil, l'action du corps laisse son empreinte dans la matière du monde sensible, faisant ainsi lieu à une certaine mémoire de son geste.

9 La perception sensorielle donne au corps accès au sensible et met en place un autre système mnémotechnique d'écriture. La vue, l'odorat, l'ouïe, le toucher, le goût permettent au corps de prendre connaissance du monde sensible environnant, en pouvant l'apprivoiser et s'y adapter. Ils circonscrivent le lieu dans lequel le corps évolue et se positionne. Ils établissent la mémoire d'un corps situé dans un contexte matériel. Avec la perception sensorielle, le corps instaure une autre catégorie de techniques, celle d'instrument. En effet, au lieu d'utiliser l'action pour la faire aboutir, comme dans les techniques d'outil, le corps va prolonger la fonctionnalité du sens de perception en recopiant ou en améliorant son organologie. L'instrument d'optique en est un parfait exemple. Ces techniques d'instrument ont un impact sur la connaissance du monde sensible. Le corps n'est plus circonscrit dans le lieu défini par ses sens, il peut jouer avec ses limites, en allant au delà de sa capacité perceptive. Les techniques d'instrument ouvrent le sensible là où la perception humaine s'arrête ${ }^{7}$. La connaissance, l'apprivoisement et l'adaptation à un environnement extérieur se délocalisent alors vis-à-vis du lieu même du corps.

La relation du corps aux sens de perception ne réduit pas les techniques d'instrument à un positionnement dans un contexte environnant. Elle possède une autre particularité plaçant leur système mnémotechnique dans le champ de la saveur. En effet, les sens permettent au corps de révéler une subjectivité par la sollicitation et l'affirmation d'un goût unique, personnel. L'enjeu de la perception, à travers ses techniques d'instrument, n'est plus de remplir une fonctionnalité mais de se servir de cette dernière pour gratifier le sujet d'un plaisir sensoriel. Le champ de la saveur s'ouvre alors à l'art de la contemplation. Prendre plaisir à voir, à entendre. Savourer le sensible pour mieux le regarder, l'écouter... Faire apparaître une certaine forme de beauté, celle issue d'une esthétique, d'une esthésie des sensations éprouvées par le plaisir sensoriel que procurent la saveur, le contact avec le sensible, et garder en mémoire toutes ces impressions.

11 Le champ de la saveur exerce en effet la subjectivité par la pratique des techniques d'instrument, où l'expression du corps, à travers ses gestes, est sollicitée. L'art s'oriente vers des techniques artistiques comme le théâtre, la musique, la danse, les arts plastiques, vers le jeu qui associe la fonctionnalité de la perception à la subjectivité. La personne, par son jeu artistique, pose son empreinte dans la matière sensible du monde. La saveur se base autant sur les mécanismes d'impression que d'expression du corps. Elle les intègre dans un même système mnémotechnique d'instrument, où la grammatisation a le jeu comme langage. Jouer avec les limites sensibles du monde ${ }^{8}$, du corps pour en faire lieu de mémoire.

12 À la suite des techniques d'instrument issues de la saveur, le corps met en place un système mnémotechnique lié au principe même de l'écriture. Plus que du graphe, 
l'écriture relève du gramme. Avant d'être manifestée par l'expression du corps, du geste, elle provient intrinsèquement d'enregistrement d'impressions.

Cette capacité autonome de production correspond à des techniques d'appareil qui ont la particularité de pouvoir reproduire et d'améliorer un fonctionnement tirant son origine de l'organologie du corps. Ce fonctionnement établit une grammatisation, voire un programme, car il équivaut à un enregistrement. En chaque gramme, il spatialise un continu temporel en une limite définie, une durée. En chaque gramme, l'enregistrement provoque des discontinuités en imprimant la matière. Formée, cette dernière contient alors toute l'information nécessaire à la reproductibilité, caractéristique essentielle de l'enregistrement. Aussi, ce serait une erreur de penser les techniques d'appareil en dehors de matérialité, et surtout pour celles du mode virtuel, numérique, car le gramme est une forme définie. C'est bien grâce à la plasticité de la matière que l'écriture peut avoir lieu. Les techniques d'appareil forment une mémoire dont le langage est de l'ordre de l'apprentissage.

14 Les techniques d'appareil appartiennent au champ du savoir. Nous distinguons le savoir de la connaissance dans la mesure où le sens du langage mis en œuvre peut échapper à la raison, la compréhension ou la volonté. En effet, ce système mnémotechnique d'appareil concerne des processus pouvant être indépendants de la maîtrise du corps, notamment quant aux fonctionnements vitaux: respirer, manger... relevant d'automatismes. Grammatisant leur langage comme apprentissage, les techniques d'appareil procèdent d'impressions. Elles constituent une mémoire sans cesse en formation, creusée, formée dans la plasticité de la matière même du corps, et où l'oubli a aussi toute sa place.

Cette matière façonnée d'impressions est une latence. C'est une mémoire présente mais inaccessible en soi. Elle est une puissance porteuse de sens, un potentiel à révéler, à exploiter par le développement de son langage, par l'entrée de l'apprentissage dans le champ de la connaissance. Notons que les fonctionnements psychique et photographique répondent aux techniques d'appareil, car ils ne sont pas seulement des instruments optiques, ils se caractérisent aussi par leur capacité d'enregistrement.

\section{Du commun de la forme vers un emboîtement d'une topologie d'inconscient}

Les techniques récupèrent chacune, quant à leur processus, une particularité organique : l'outil utilise l'action, l'instrument prolonge la fonctionnalité et l'appareil reproduit le fonctionnement. Elles ont en commun d'être capables d'écriture, en développant chacune une grammatisation d'un langage spécifique à un champ. Respectivement, le geste, le jeu et l'apprentissage viennent constituer la mémoire du savoir-faire, de la saveur et du savoir, en la définition d'une forme.

La forme, c'est la mémoire du lien physique d'une action sur une matière. Avant d'être une particularité organique, le processus des techniques d'outil, d'instrument et d'appareil correspond alors au tracé, principe d'écriture. Il donne forme à la matière en posant une action. Suivant qu'il se situe dans les mécanismes d'impression ou d'expression du corps, il révèle une plasticité manifeste ou latente des systèmes mnémotechniques, plus ou moins évidente selon la force de l'action et la résistance de la matière. 

à à l'infini, ni dans l'étendue ou le flux d'une continuité, mais au contraire dans le défini et la discontinuité spatio-temporelle du tracé. La rupture, la limite du cerne se fait donc entre l'arrêt et le flux, le défini et l'infini, la discontinuité et la continuité. Mais la forme ne se résume pas à cette rupture qui distingue et sépare, elle correspond davantage à la corrélation d'un arrêt dans le flux, à une brèche du défini dans l'infini, à l'ouverture d'une discontinuité dans la continuité. Séparer pour relier ; la forme structure le lieu de l'inconscient tel un tissu. La topologie des systèmes mnémotechniques d'écriture s'apparente à des surfaces tramées, des lieux structurés faits de plein et de vide, d'entrelacs et d'interstices, com-posés de points de capiton ${ }^{10}$ sur la surface de la matière.

Si la structure du lieu d'inconscient relève d'une discontinuité dans la continuité, comme tissu, elle révèle une continuité de discontinuités. La structure concerne aussi la réitération de la forme, en étant capable de re-faire des arrêts dans les flux, dans la matière. La structure est le moyen par lequel le fonctionnement d'inconscient développe son lieu et le sens qui va avec. Elle confirme de ce fait que l'inconscient, comme système mnémotechnique, produit de la grammatisation. En effet grammatiser, c'est écrire à partir de la reproductibilité de chaque élément d'un système. C'est construire du texte à partir d'un alphabet, où le sens prend forme par l'association des lettres, en l'enchaînement distinct de mots et de phrases. La structure, au sein du tracé comme processus d'écriture, réitère la forme en procédant du défini de la lettre vers l'indéfini du texte. L'indéfini consiste à dépasser la définition, la limite, le cerne, sans 
jamais l'annihiler. C'est pourquoi la topologie d'inconscient ne peut se confondre avec les flux de l'infini ni se cantonner à l'arrêt de la définition de la forme. Elle correspond à l'indéfini d'un texte qui n'est autre qu'une juxtaposition successive de lettres dans laquelle s'emmaille le sens. L'indéfini ouvre le texte sur une polysémie qui gagne en épaisseur. En effet, en dis-posant les lettres les unes à côté des autres, l'indéfini étoffe, compose le sens tel un rébus. Mais pour accéder au sens, il faut aller le chercher dans la forme.

Les processus d'outil, d'instrument et d'appareil, en répondant au tracé, comme principe d'un système mnémotechnique, produisent une écriture structurée selon une réitération de formes qui se répercute tout autant dans le savoir-faire, la saveur et le savoir. Par déduction, ces trois champs possèdent une topologie d'inconscient ouverte par la forme. Au commun de cette topologie, peut-on sup-poser un emboîtement de ces trois registres d'inconscient, de leurs textes ? Peut-on supposer que le geste exprimé du savoir-faire soit en résonance, tel un écho, avec l'apprentissage imprimé du savoir ? Ya-t-il une super-position de langages?

La superposition des champs et de leurs langages va de pair avec l'emboîtement des techniques respectives. Cela signifie que le fonctionnement des techniques d'appareil s'emboîte dans l'action des techniques d'outil. L'emboîtement trouve alors son origine dans le corps. En effet, toutes les techniques dépendent du corps et se différencient en catégories suivant qu'elles utilisent, prolongent ou reproduisent les processus d'action, de fonctionnalité ou de fonctionnement. L'emboîtement se situe au niveau de la catégorie d'instrument, des techniques qui font passer, par le jeu, l'apprentissage dans le geste et réciproquement.

Le jeu est un langage qui appartient au champ de la saveur, et dont la particularité est de solliciter les mécanismes d'impression et d'expression du corps. Il donne accès au sensible, en jouant sur les limites d'un contexte tout en permettant l'empreinte d'une subjectivité. Le jeu fait passer ${ }^{11}$. Il permet d'imprimer ou d'exprimer les formes. Et pour ce qui est de l'emboittement de l'apprentissage dans le geste, le jeu fait passer, en exprimant, le savoir dans le savoir-faire. Le langage de la saveur s'implique alors dans le champ du faire et mobilise le corps dans un pouvoir-faire. Il s'agit de chercher la concrétisation d'un potentiel par l'engagement du corps. Par le pouvoir-faire relatif à l'effort du faire, le geste devient l'expression d'un apprentissage. Il est l'acquisition d'une mémoire, ouvrant par conséquent sur une réitération des formes manifestées dans le sensible. Le jeu est l'exercice d'un re-faire, qui ne doit pas être assimilé à une répétition. Dans ces conditions, la grammatisation du faire, du pouvoir-faire définit le jeu comme le langage d'un programme, où la manifestation des formes n'est que l'expression d'une pré-inscription, faisant ainsi perdre au texte son sens, son orientation.

Pour cette raison, les champs du faire et de la saveur sont indissociables. Ils possèdent le même langage du jeu, tout en le développant chacun avec ses propres spécificités. La saveur apporte au jeu l'envie et le plaisir de faire par le moyen de la gratification sensorielle. Le jeu mobilise le corps à rechercher l'objet de son aspiration, de ce qui lui procure la satisfaction du sens. Il y a alors élaboration du désir comme un tendre vers, soit une projection - l'ouverture possible d'un espace de temps correspondant à la définition du gramme. Cependant si le jeu est juste tenu à assouvir un plaisir immédiat, à répondre à une pulsion, sans désir ni satisfaction du faire, il est sans saveur. Il n'est même plus langage, car il ne s'intègre plus dans un processus de grammatisation. Il a 
perdu la structure à l'origine de la réitération des formes permettant la construction d'un texte manifestée notamment par l'évolution de l'être humain et de son lien social $^{12}$.

En mobilisant le jeu par le désir, la saveur est en quête de l'affirmation du goût. Elle produit un langage révélant une subjectivité. La saveur fait appel à ce qui n'est pas encore factuel, im-primé dans la mémoire, mais possible grâce à l'imagination du jeu. Ce dernier fait passer le savoir-faire dans le savoir. Le langage du geste ${ }^{13}$ s'emboîte dans celui de l'apprentissage. L'imagination du jeu consiste à re-faire des formes sensibles, tel un recommencement qui engramme une nouvelle mémoire, participant à la progression indéfinie de sa topologie. Le texte du savoir prend sens, en ayant une écriture qui avance, qui se projette.

Le jeu de l'emboîtement, qu'ouvre la saveur, est ce par quoi passent l'expression de l'apprentissage dans le savoir-faire et l'impression du geste dans le savoir. Le jeu repose aussi intrinsèquement sur le pouvoir et l'envie de faire de la saveur dans l'emboîtement d'un vouloir-faire. Il mobilise ainsi l'intégralité psychique et physique du corps ${ }^{14}$, d'un être corps. Située dans le champ de la saveur, l'art-thérapie est une méthode de soin par l'art et non avec l'art, interpelant le vouloir-faire du corps. Au moyen de l'art-thérapie, le corps peut alors jouer à emboîter, comme des poupées russes, ses impressions et expressions dans une superposition des langages respectifs.

L'emboîtement est un jeu de superpositions grâce à la saveur qui s'avère être le champ caractéristique du corps. C'est elle qui le place, comme être vivant animé, à l'origine des différents systèmes mnémotechniques. Le jeu de la saveur est ce par quoi le corps est capable de trouver l'essentiel de l'emboîtement, à savoir la pose, la pose de l'action sur la matière correspondant à la formation même du gramme, sa forme. Il confirme donc l'emboîtement des techniques, catégorisées selon leur application dans les champs, dans l'intégration du fonctionnement d'appareil au sein de l'action d'outil. Seulement le fonctionnement d'appareil relève d'automatismes, il échappe à la maitrise du corps. Cela ne remet pas en cause l'origine de sa technique, car le corps a la capacité, dans son vouloir-faire, d'enclencher et de déterminer la durée de la pose. Le corps choisit, décide le début et la fin de l'action. Il permet à la pose d'être une forme définie selon un espace de temps et de devenir ainsi un lieu de mémoire, un gramme pouvant être aussi bien im-primé qu'ex-primé.

30 La pose est le sens même de la forme. Elle fonde tous types de relations qui se déclinent à travers de ce qui dé-pose, re-pose, im-pose, ex-pose, dis-pose, com-pose, ap-pose, oppose, sup-pose, super-pose... En effet, ne concernant rien d'autre que l'action sur une matière, la pose est cette forme initiale qui vient entre l'être humain et le monde : cette pression entre eux s'exerce à travers la force et la plasticité respectives de ce qui est de l'ordre de l'im-pression, ex-pression, dé-pression, ré-pression, sup-pression, oppression... Et le travail de l'art-thérapie est justement de chercher cet initial de la forme pour y interroger la pose, la pression.

\section{D'une pratique d'art-thérapie}

31 Cette pose, cette pression permettant au sein du corps l'emboîtement des systèmes mnémotechniques dans la résonance de son jeu entre l'expression du geste de son savoir-faire et l'impression de l'apprentissage de son savoir, est pour l'art-thérapie un idéal; ce qui définit son travail, son enjeu, ce sur quoi, en tant que méthode 
thérapeutique, elle doit agir et être efficace. L'art-thérapie travaille sur trois axes qui constituent justement la pose du tracé, à savoir l'action, la matière et la structure.

L'action, tout d'abord, est indissociable de la pratique art-thérapeutique, car elle joint la cohérence d'un processus thérapeutique à une opération artistique. L'action permet au corps d'agir, d'œuvrer, de faire œuvre, de produire. L'action est le fondement de la méthode art-thérapeutique, puisqu'elle implique le champ de la saveur dans le faire du corps. Être dans l'action, c'est faire. L'action mobilise le corps et le désigne comme origine d'un faire-œuvre.

L'opération artistique n'est pas menée en fonction de la finalité de l'œuvre. Elle ne renvoie pas aux codes de la représentation, où le corps, instrumentalisé par la technique, est tenu à distance de son action afin de répondre à une logique de cadre. L'art-thérapie ne réfère donc pas l'action entreprise aux catégories de l'icône ou du symbole de la trichotomie peircienne. En plaçant le faire-œuvre dans le principe du tracé, l'action n'a pas à produire une ressemblance ou une similarité d'objet situé, de surcroît, en dehors de la nécessité d'existence et de lien avec un référent physique. La pratique de l'art-thérapie se rapporte à l'index. Elle amène l'action à produire des formes et à les rendre manifestes quand elle les inscrit par le geste du corps œuvrant.

Travailler sur l'action permet à l'art-thérapie outre de définir le corps comme origine d'une production manifeste de formes, de révéler sa subjectivité. Dans ces conditions, l'art-thérapie peut influer sur la relation de la personne avec le monde extérieur. Le champ de la saveur prend ici toute son importance en faisant passer les in-formations grâce aux capacités sensorielles, sensitives du corps baigné dans le sensible du monde. $\mathrm{Au}$ moyen de l'action, ces informations vont alors se former, prendre forme à travers respectivement l'impression du monde et l'expression du corps œuvrant et pouvoir viser l'emboîtement des langages du savoir-faire et du savoir. Cet emboîtement se répercute au sein même du faire-œuvre qu'est l'opération artistique. En effet, chaque technique artistique se base sur les mécanismes d'impression et d'expression du corps en développant conjointement, par l'action, contemplation et pratique. Il faut savoir écouter pour jouer, regarder pour peindre, c'est-à-dire sentir pour agir, mêler la saveur au faire. L'art-thérapie s'appuie sur cette articulation artistique. Elle devient processus thérapeutique quand elle vient remédier à une défaillance empêchant la cohérence de cette articulation de la pression qui pose l'origine de la relation entre le corps œuvrant et le monde. Cette défaillance se résume généralement à une action qui ne produit ni d'impacts ni formes.

Dans le processus art-thérapeutique, l'action permet de travailler sur l'engagement du corps œuvrant, en le mobilisant notamment à travers l'expression de son geste. En effet, l'art-thérapie fonctionne à la mesure de l'engagement de la personne, de son aptitude et sa volonté à se placer dans le champ du faire, le faire-œuvre, à se définir comme l'origine de ce qui se fait, s'œuvre afin d'être en lien avec le monde extérieur. L'engagement c'est être pleinement dans l'action afin de pouvoir se confronter à l'expérience de la responsabilité. La corrélation peut sembler évidente, puisque la responsabilité provient littéralement de l'engagement. Son étymologie "respondeo » signifie répondre de, être garant. L'engagement prend et fait poids dans la responsabilité qui, en son nom res pondere, détient les choses lourdes. L'engagement conduit à la responsabilité et la responsabilité traduit l'inscription de l'action. Être responsable, en s'engageant, permet alors de pouvoir inscrire son action sur la matière, y poser son poids. 

effectives que lors d'une confrontation au monde extérieur. Le jeu s'affine et se précise dans un apprivoisement du monde et une adaptation du corps au monde, en aidant le corps à trouver les lois et à y obéir. L'engagement et la responsabilité amènent le corps œuvrant à être dans l'obéissance ${ }^{15}$ des lois, condition nécessaire pour pouvoir se structurer. En l'occurrence, la loi n'est pas à confondre avec la norme ou la codification, qui entraînent une soumission au lieu de l'obéissance. Répondre à la norme empêche de se penser comme origine de l'action menée. C'est se soumettre à des codes sociaux, culturels, représentatifs, en produisant des signes qui, au niveau d'un processus artistique, répondent aux catégories de l'icône ou du symbole. De la soumission découle le risque de la ré-pression ou l'op-pression, pouvant se traduire par une attitude contenue ou retenue du corps. Et tout le travail de l'art-thérapie sur l'action, pour qu'elle produise des signes définis selon des formes, consiste à faire éprouver au corps cette différence entre loi et norme. Le travail sur l'action étoffe le «prendre soin » de la méthode art-thérapeutique. Il s'agit d'aider la personne par son faire-œuvre à s'affirmer en se confrontant à des limites, à pouvoir se définir et mieux se connaître, à gagner donc en confiance et en estime de soi.

L'enjeu de l'action est de rencontrer la matière pour y produire un impact, une pression, une pose. Tout autant que l'action, la matière est indispensable pour le travail des formes en ce qui concerne leur composition. La matière reçoit l'impact et en com-pose la forme. Originellement, la matière peut être sans forme, puisqu'elle est assimilée aux flux. Et le travail des formes consiste à presser cette matière, par l'action mise en œuvre, en jouant avec sa plasticité. Grâce aux formes, la matière absorbe de la subjectivité, et tout par conséquent acquiert de la matière, comme les idées, les sentiments, les émotions, l'oubli... Ne dit-on pas qu'une tension est palpable ? La matière concerne bien la com-position des formes. Elle est indissociable de la pose mais sans la produire : elle est avec. Elle porte la marque, l'empreinte. La catégorie des signes de l'index ne peut se concevoir en dehors d'une matérialité. Il en est de même pour les systèmes mnémotechniques d'écriture. L'emboîtement de leurs champs d'application et langages est possible grâce au faire du jeu, car ils ont en commun d'être des systèmes matériels

tion interpelle le pouvoir-faire du corps, l'ensemble du champ du faire se mêle à celui de la saveur. Et la saveur a la particularité de solliciter la catégorie d'instrument 
pour gratifier la sensorialité du corps. Elle mobilise, de ce fait, l'envie de faire du corps, qui n'est autre que l'accès à la matière. La forme est une action posée sur la matière. Elle relève d'un tact avec elle, elle est un contact. La gratification sensorielle consiste à trouver ce contact. Elle se rapporte donc au tactile, voire au gustatif inhérent à chaque sens de perception. L'envie de faire, qu'ouvre la saveur, peut aller au-delà de la simple notion de plaisir, elle est davantage une appropriation de la matière. Produire une forme, c'est toucher la matière, la savourer, se l'approprier, la manger en l'ingérant et la digérant pour la faire sienne, et se constituer, par sa com-position, une topologie d'inconscient, un lieu de mémoire propre à chacun. L'appropriation de la matière amène à se considérer soi-même comme matière et d'accepter que tout soit matière, $\mathrm{y}$ compris ce qui n'est pas manifeste. Certes, la topologie d'inconscient relative au champ du savoir, n'est pas phénoménologiquement visible mais elle peut être lisible, car tout processus technique produisant de l'écriture s'appuie sur une existence matérielle. Ce lieu de mémoire constituant notamment le savoir est une matière, dont le travail des formes révèle qu'elle est com-posée par une grammatisation procédant au langage de l'apprentissage.

41 Accepter et s'approprier la matière permet à l'action de s'inscrire en produisant du plein, d'y creuser, d'y graver des formes. L'instant, qui est la définition de la forme dans la durée de la pose, ouvre au corps œuvrant le langage du jeu et lui permet de faire l'expérience du plein. Si le plein est matière, la réciproque n'est pas forcément de mise quand cette dernière est assimilée aux flux. En effet, le plein se rapporte à la composition de la forme prenant lieu au sein de l'instant. C'est pourquoi être dans l'instant, c'est l'habiter, c'est y être pleinement en dehors de toute sensation de vide. La composition des formes montre l'importance de consentir à la plasticité de la matière : la plasticité est à la matière ce que la force est pour l'action. Elle est la condition nécessaire à l'élaboration de la pression. Elle est la caractéristique permettant à la matière de supporter la forme, comme le plein du contact avec l'action, dont le creux de la trace, de l'empreinte en garde la mémoire.

En œuvrant, le corps s'approprie donc la matière et s'accepte en tant que tel. Il se place alors dans le champ de la saveur, c'est-à-dire dis-posé à la saveur. Seulement, qui dispose de cette matière et comment dispose-t-on d'elle ? La disposition de la matière provoque souvent la négation, voire l'annihilation des formes, car elle est à l'origine du dysfonctionnement de la pose et du désemboîtement des systèmes mnémotechniques d'écriture. Le fonctionnement d'appareil, malgré l'action, n'arrive plus à définir une ouverture d'instant en ne trouvant plus de surface à grammer. Le refus de la plasticité concerne ce dysfonctionnement relatif à la disposition de la matière. Il s'exprime dans une alternative : déni ou vide de la matière. Le déni de la matière modifie la plasticité de sa surface en une élasticité. Rien ne s'accroche, rien ne se gramme. Résulte une difficulté, voire une impossibilité d'impression et d'expression. Tout reste lisse, hermétique. Les flux se regonflent et redeviennent intacts. Quand le corps refuse la saveur par déni, même s'il est œuvrant, même s'il se place dans le champ du faire en activant son pouvoir-faire, il entraîne la sup-pression des formes. Le refus de la plasticité comme vide de la matière correspond, quant à lui, à une non rencontre de la surface. Le vide révèle alors un problème de consistance même de la matière. Il amène le corps œuvrant à éprouver l'expérience de la non-matière, autrement dit à être dans l'épreuve de la dé-pression. 

notamment sur la plasticité, et plus précisément sur ses propriétés que sont la résistance et la consistance. En effet, quand le corps est dans le déni de la matière, l'artthérapie a pour objectif de lui faire accepter la saveur en cherchant le ressenti. Il faut placer l'action dans le champ de l'esthésie pour favoriser la sensation et l'impact qu'elle peut procurer par une certaine quête du plaisir. L'impact est à associer au goût, à la saveur et par là même à la matière, qu'il faut donc s'approprier pour pouvoir ressentir le plaisir de la sensation que génère l'action. La résistance s'énonce dans cette quête même du ressenti, sans cesse aller le chercher là où la matière va prendre corps. Cela permet de s'extirper du déni. La résistance permet l'impact de la sensation, qui rencontre la plasticité de la matière et non son élasticité. Les formes ne sont alors plus supprimées. En ce qui concerne le vide de la matière, l'art-thérapie doit entreprendre un travail sur sa consistance. S'appuyer sur l'action pour révéler que le vide n'est pas une non-existence matérielle, mais qu'il équivaut davantage à une dilatation de matière empêchant toute accroche des formes. L'action doit être répétitive. Son impact, aussi insignifiant, imperceptible, infime soit-il, à force d'être déposé, engendre par sa multiplication l'épaisseur d'une empreinte. Il constitue ainsi une densité de la matière. Il est possible, pour le corps, de quitter la déprime voire la dépression en trouvant, par la superposition des déposes de son action, une pression des formes. la gravité soulignée par la pression. Graver la forme, c'est faire l'expérience de la chute sans perdre pied. La plasticité retient le corps œuvrant de tomber dans un vide tel une béance sans fond, car l'art de faire des formes équivaut à creuser ${ }^{16}$, à marquer la matière sans la percer, sans la trouer mais bien de modeler sa surface. En acceptant la responsabilité du geste, le corps obéit à la gravité et laisse choir le poids de son action sur la matière. La plasticité autorise l'obéissance à la loi, qui en l'occurrence s'avère être l'association de la responsabilité à la gravité et à laquelle répond la pression afin de pouvoir produire des formes. La matière reçoit l'action. À travers l'expression du geste, elle l'absorbe, tel le sol le pied du danseur pour mieux le faire rebondir. La matière est comme un ressort qui engendre de l'action dans le rebond. En mêlant l'envie de faire, ou plus exactement un saveur-faire au pouvoir-faire du corps, la matière engendre l'action, car par le rebond il est bien question d'un re-faire des formes selon un vouloirfaire. Elle permet le pouvoir-fonctionner d'un système mnémotechnique. La structure procède alors d'une grammatisation relevant d'une catégorie technique d'appareil capable d'écriture. Au moyen du gramme, elle participe à l'élaboration d'un texte par la définition de la lettre. Dans une approche plus factuelle, la structure correspond au cadre du travail art-thérapeutique. Elle se retrouve notamment au sein du lieu de l'atelier où elle distribue et compartimente la spatialité. Chaque endroit de l'atelier a une fonction précise qui va de pair avec la dynamique de la séance. Structurer l'espace permet de poser des limites formelles tels des rituels immuables et communs à toutes les séances.

46 La dynamique correspond à l'ensemble des différentes phases composant une séance. Elle se répercute dans n'importe quelle technique artistique, notamment les arts plastiques ou le théâtre. Entre l'accueil et la discussion, la dynamique consiste dans la préparation des couleurs et l'action; elle marque une étape d'exploration, de 
connaissance et de jeu. Caractéristique de la méthode art-thérapeutique, la dynamique incorpore la scansion et la structure du cadre dans une progression temporelle. Chaque phase est tenue selon un ordre, un déroulement précis ayant comme enjeu la manifestation d'une production de formes, en faisant évoluer l'action du geste et l'implication du corps œuvrant au rythme d'une logique d'enchaînements de sollicitations spécifiques. La structure s'applique tant dans l'espace que dans le temps et répond à la fonction qui consiste à provoquer une continuité de discontinuités spatio-temporelles. La structure permet donc le pouvoir-fonctionner en reliant ce qu'elle distingue.

La structure participe à la pression de la forme en condensant l'association de l'action et de la matière. Pour marquer une progression spatio-temporelle, elle doit canaliser l'action dans le mouvement du geste et trouver la plasticité de la matière. La structure octroie au mouvement d'extraire la force de l'action et fournit à la plasticité le rebond de la matière, éléments fondamentaux de la pression. La structure combine le rebond de la force et la force du rebond, dont la particularité est de loger la pression dans la définition du gramme. En effet, la pression devient une réaction.

La réaction confirme que la pression est bien un gramme, car elle a une valeur d'enregistrement. Elle est la trace portant la mémoire d'un acte. Sa forme peut être reproductible. Réduite à l'essentiel, elle est un élément d'une grammatisation élaborant tout principe de langage. L'enjeu de la structure est la capacité à ré-écrire des lettres, à re-faire donc, sans omettre l'avancée du texte. La réaction que provoque la structure extirpe le gramme d'un programme et permet à la forme de ne pas être formatée, car alors le rebond ne ferait que du surplace. La forme serait alors prisonnière d'un cercle fermé dont elle ne pourrait sortir, condamnée sans cesse, malgré la structure, à être la répétition d'un même, une mémoire tournée sur elle-même, se dé-maillant de toute histoire.

La réaction révèle que le rebond de la matière génère une nouvelle force d'action. Elle la projette en avant afin qu'elle produise dans l'ouverture d'un espace de temps, dans la durée d'une pose, une nouvelle pression. En tant que gramme, cette dernière s'avère être semblable à ce dont elle est issue mais pourtant autre, car porteuse d'un sens encore à faire, à découvrir. La structure laisse place à l'imagination. Elle la fait cohabiter avec la mémoire au sein du gramme, plus exactement en la réaction de la pression. Re-faire, ré-écrire des lettres est un éternel recommencement. La structure fait passer le gramme du défini vers l'indéfini où de la lettre au texte la grammatisation s'énonce selon un alphabet. La topologie d'inconscient comme lieu de mémoire provient bien alors d'une typologie qui lui porte sens (sens dans l'ouverture d'une signification). En effet, tout langage fondé sur une grammatisation a une visée communicante. Il cherche un échange d'informations. Il y a l'émergence, par la forme signifiante, d'un contenu signifié dont peut s'échapper toute une polysémie. En s'appuyant sur la réaction de la pression, la structure fait rebondir le sens, l'amenant à quitter la forme, à s'en émanciper, pour être jeté dans une apesanteur. C'est l'expérience du Sublime.

50 Le Sublime répond au pouvoir-fonctionner de la structure, à savoir provoquer une continuité de discontinuités. Le Sublime est une confrontation à une limite qu'il faut dépasser sans l'annihiler par un changement d'état matériel. Telle est sa signification notamment étymologique et commune aux domaines esthétique, psychologique et physique. Le Sublime ne se situe pas en dehors de la matière. Il est un accès à un autre 
état, où l'apesanteur n'est pas un vide mais un état gazeux, une sorte d'appel aérien, juste le temps d'un rebond annonçant une chute. Quand la structure fait passer du défini vers l'indéfini de la forme, elle amène le sens à se mouvoir du sensible perceptif, orienté, vers une signification où il se recoupe entièrement dans les changements d'état de la matière. Le Sublime, c'est donc du vertige, le vertige du sens en un jet dans l'inconnu.

Dans le vertige du Sublime, ce jet, intrinsèque à l'action/réaction, est à la fois dénué, libre de ce qui le pré-pose, d'être (ob) (su) (pro)-jet et lourd, responsable pour (re) tomber sur la surface de la matière, afin de ne pas se dissiper dans l'infini des flux.

Le Sublime ouvre à l'aérien par l'oral et fait accéder au langage verbal en tant que parole. Au delà des besoins vitaux, la parole est ce qui caractérise l'humanité de l'être vivant. En effet, l'être humain est doué de parole. Il parle. Sa voix émet des sons propulsés dans l'aérien signifiant du sens. Il dit des mots. Seulement, la voix n'était-elle pas initialement une langue qui s'agite dans la bouche, un langage gestuel issu de la catégorie technique d'outil? Mais lequel s'il n'était pas pris dans le pouvoirfonctionner de la structure ne serait qu'une gesticulation vide de sens. La voix ${ }^{17}$ est le lien entre la parole et le corps.

La voix est ce verbal, qui à l'instar du verbe est l'action mouvant la phrase dans l'aérien, sublime, puisque manifestée par le corps. Elle est ce geste de parole. En effet, la voix, à perdre corps, serait dépourvue de structure lui permettant d'être jetée. Elle serait uniquement prise dans la catégorie technique d'appareil, réduite à prononcer le dernier mot sans pouvoir l'initier, tel l'écho... Écho... Le sens s'éparpille oralement à l'infini, dans les flux. Pour se transmettre pleinement, le sens doit s'inscrire à travers le poids des formes, à travers les systèmes mnémotechniques d'écriture. Le travail artthérapeutique sur la structure consiste à incorporer le sens au sein des formes jusqu'au Sublime pour être jeté, libre et responsable, aérien et lourd, dans le vertige. Plein, le sens répercute l'ensemble de ses définitions dans les langages du geste, du jeu et de l'apprentissage, emboîtant les systèmes mnémotechniques. Il peut ainsi faire tourbillonner la parole entre l'oral et l'écrit.

Tout art est capable de Sublime, en faisant rebondir le sens grâce à une mise en œuvre des formes. La structure est inhérente à chaque technique artistique. Elle se comprend sous le dénominateur commun d'un tempo et propre à la fois à la poésie, à la danse, à la musique, au théâtre, aux arts plastiques... Ce tempo ne marque pas la régularité d'une mesure, mais une continuité de discontinuités dans l'association tourbillonnante de la pression et du vertige, tel un "danser de l'art » où le sens prend corps, fait corps, devient corps œuvrant à travers l'emboîtement des systèmes mnémotechniques d'écriture.

Mais avant toute technique artistique, ce tempo, comme pouvoir-fonctionner d'une structure, est intrinsèque à la racine du corps, c'est-à-dire l'être vivant de l'être humain. Le tempo premier est la respiration. Le souffle ${ }^{18}$ inspirant et expirant répond à une organologie fonctionnant sur l'automatisme d'appareil qui se manifeste par la force de l'action. Il est cet art faisant que le corps devient une logique même de processus d'écriture, tissant son propre texte dans l'étoffe d'un contexte, et où l'artthérapie doit chercher les fils dénoués ou emmêlés d'une trame afin que le sens puisse avancer selon l'ouverture du désir.

Située entre la technique et le soin, l'art-thérapie aide le corps, au moyen d'un « danser de l'art ", d'un tempo, à faire des formes, à produire du texte sur des matérialités 
manifestes ou latentes qui s'emboîtent, comme des poupées russes, au sein d'un fairelieu et dont l'en-jeu est d'assimiler, dans une topologie d'inconscient, le lieu de mémoire au champ de l'imaginaire. L'art-thérapie est donc, par l'art du geste, une thérapie du faire. Elle cherche à placer le corps œuvrant dans une économie d'un faire-jeu, afin qu'il accède à l'imaginaire, car ce dernier est le champ où la subjectivité germe grâce au potentiel d'un vouloir-faire. L'imaginaire ${ }^{19}$ s'avère être le berceau du désir.

L'imaginaire est accessible par d'autres portes, comme la sexualité, le rêve, l'espoir... qui tous cherchent à faire lien avec la réalité à travers la quête corrélative du sens et du désir. L'art-thérapie n'a pas intérêt à s'enfermer dans une application finie d'une méthode programmée mais à se construire, à s'emboîter indéfiniment au sein d'une transdisciplinarité, ce qui lui permettra de déployer la compréhension du sens et de sans cesse pouvoir s'adapter et accompagner au mieux le corps œuvrant vers une économie du désir.

\section{BIBLIOGRAPHIE}

Barthes Roland, L'obvie et l'obtus, Paris, Seuil, 1982.

Benjamin Walter, « L'œuvre d'art » (dernière version), in Euvres III, Paris, Folio-Gallimard, 2000.

Benjamin Walter, « Petite histoire de la photographie », in Euvres II, Paris, Folio-Gallimard, 2000.

Castarede Marie-France, La voix et ses sortilèges, Paris, Les Belles Lettres, 2004.

Deledalle Gérard, Écrits sur le signe, Paris, Seuil, 1978.Stiegler Bernard, Prendre soin de la jeunesse et des générations, Paris, Flammarion, 2008.

Freud Sigmund, « Note sur le bloc-note magique », in Résultats, idées, problèmes, t. 2, Paris, PUF, 1985.

Guérin Michel, Philosophie du geste, Arles, Actes Sud, 1995.

Lacan Jacques, Le séminaire III, Les psychoses, Paris, Seuil, 1981.

Leroi-Gourhan André, Le geste et la parole, t. 2, Paris, Albin Michel, 1965.

Mauss Marcel, « Les techniques du corps », in Sociologie et anthropologie, Paris, PUF, 1983.

Peirce Charles Sanders, Collected Papers, Cambridge, Harvard University Press, 8 vol., 1931-1958.

Simondon Gilbert, L'individuation psychique et collective, Paris, Aubier, 1989.

Winnicott Donald Woods, Jeu et réalité, Paris, Folio-Gallimard, 2002.

\section{NOTES}

1. Stiegler Bernard, Prendre soin de la jeunesse et des générations, Paris, Flammarion, 2008.

2. Barthes Roland, L'obvie et l'obtus, Paris, Seuil, 1982, p. 146-148. 
3. Peirce Charles Sanders, Collected Papers, Cambridge, Harvard University Press, 8 vol., 3.361, 8.335, 1.558 ; Cf. Deledalle Gérard, Écrits sur le signe, Paris, Seuil, 1978.

4. Mauss Marcel, « Les techniques du corps ", in Sociologie et anthropologie, Paris, PUF, 1983, p. 372.

5. Guérin Michel, Philosophie du geste, Arles, Actes Sud, 1995, p. 25.

6. Ibid., p. 34 .

7. Benjamin Walter, «Petite histoire de la photographie », in Euvres II, Paris, Folio-Gallimard, 2000, p. 298.

8. Benjamin Walter, "L'œuvre d'art » (dernière version), in CEuvres III, Paris, Folio-Gallimard, 2000, p. 306.

9. Freud Sigmund, « Note sur le bloc-note magique », in Résultats, idées, problèmes, t. 2, Paris, PUF, 1985.

10. Lacan Jacques, Le séminaire III, Les psychoses, Paris, Seuil, 1981, p. 304.

11. Winnicott Donald Woods, Jeu et réalité, Paris, Folio-Gallimard, 2002, p. 90-126.

12. Simondon Gilbert, L'individuation psychique et collective, Paris, Aubier, 1989.

13. Leroi-Gourhan André, Le geste et la parole, t. 2, Paris, Albin Michel, 1965, p. 135.

14. Winnicott Donald Woods, Jeu et réalité, p. 110-111.

15. Stiegler Bernard, Prendre soin de la jeunesse et des générations, p. 50.

16. Guérin Michel, Philosophie du geste, p. 50-52.

17. Castarede Marie-France, La voix et ses sortilèges, Paris, Les Belles Lettres, 2004, p. 142.

18. Ibid., p. 131.

19. Stiegler Bernard, Prendre soin de la jeunesse et des générations, p. 33-34.

\section{RÉSUMÉS}

L'art-thérapie est une pratique qui cherche l'autonomie de la personne par l'art. Sa visée thérapeutique est d'aider techniquement le corps à jouer pour produire des formes. Un langage d'inconscient se révèle ainsi par le tracé que manifeste l'expression du geste.

\section{INDEX}

Mots-clés : art, art-thérapie, corps, forme, geste, gramme, inconscient, jeu, langage, mémoire, saveur, sens, technique, tracé

\section{AUTEUR}

\section{CAROLE MADELAINE-DUPUICH}

Art-thérapeute exerçant à Metz, docteur en arts et sciences de l'art Paris 1 Panthéon-Sorbonne, DNSEP des Beaux-Arts de Nantes, carole_madelaine@yahoo.fr, http://cmadelaine.arthe.free.fr, membre de l'association « à propos », http://www.aproposmetz.com 